Voix et Images

volxetimages

\title{
De la critique formaliste à la sémiologie visuelle
}

\section{Fernande Saint-Martin}

Volume 9, numéro 1, automne 1983

Guy Dufresne

URI : https://id.erudit.org/iderudit/200422ar

DOI : https://doi.org/10.7202/200422ar

Aller au sommaire du numéro

Éditeur(s)

Université du Québec à Montréal

ISSN

0318-9201 (imprimé)

1705-933X (numérique)

Découvrir la revue

Citer cet article

Saint-Martin, F. (1983). De la critique formaliste à la sémiologie visuelle. Voix et Images, 9(1), 85-95. https://doi.org/10.7202/200422ar d'utilisation que vous pouvez consulter en ligne.

https://apropos.erudit.org/fr/usagers/politique-dutilisation/ 


\title{
De la critique formaliste à la sémiologie visuelle
}

\author{
par Fernande Saint-Martin, Université du Québec à Montréal
}

L'ampleur des développements récents des sciences humaines, notamment de la psychologie et de la linguistique, permet d'entrevoir la constitution prochaine d'une véritable sémiologie du langage visuel. Cette sémiologie se proposerait d'articuler les éléments premiers constituant les signifiants plastiques, de définir, en deuxième lieu, un système modélisant de leurs interrelations syntaxiques et finalement, de préciser leur ouverture sémantique propre.

Cette démarche sémiologique exige cependant, comme préalable, la remise en question de certains éléments de la critique formaliste proposée par Clement Greenberg, en particulier de la notion de "planéité de la surface» et ses rapports avec le fait pictural. L'hypothèse d'une bidimensionnalité possible dans le phénomène pictural soulève, d'une part, la problématique de la perception/construction d'un espace et d'autre part, celle des représentations géométrales et picturales de la spatialité. ${ }^{1}$

Rappelons d'abord les commentaires de Gleizes et Metzinger, en 1912, sur le Cubisme analytique :

Si l'on devait rattacher l'espace des peintres à quelque géométrie, il faudrait référer aux savants non-euclidiens, méditer longuement certains théorèmes de Riemann. ${ }^{2}$

Il est manifeste que cette «méditation» n'a pas été véritablement entreprise par l'histoire de l'art ou la critique d'art, en dépit des incitations répétées de Pierre Francastel à cet effet. ${ }^{3}$ De façon générale, l'expression

1. Cf. SAINT-MARTIN, Fernande, Structures de l'espace pictural, Montréal, HMH Hurtubise, 1968.

SAINT-MARTIN, Fernande, les Fondements topologiques de la peinture, Montréal, HMH Hurtubise, 1980.

2. GLEIZES, A., et METZINGER, J., Du Cubisme, Paris, 1912.

3. FRANCASTEL, Pierre, «Espace génétique et espace plastique» la Revue d'esthétique, 4, 1948, pp. 349-380. 
"géométrie non-euclidienne», ou «espace non-euclidien», a plutôt été retenue comme une sorte d'étendard, de symbole vague, un signe quasi poétique, d'une volonté de transformation des structures spatiales, sans que l'on se préoccupe de relier cette formule à l'évolution réelle des structures de l'espace pictural.

Il en est ainsi dans les quelques textes accessibles aujourd'hui du Formalisme russe qui tentera, entre 1915 et 1925 , d'élaborer les principes de description du nouvel objet pictural issu du Cubisme. Pourtant la critique formaliste russe était favorablement située pour réaliser une prise de conscience plus poussée, étant donné l'impact qu'avaient eu, en Russie, les recherches fondamentales de Lobatchevsky et de Riemann, comme nous le signale Nikolaï Taraboukine. ${ }^{4}$

À maintes reprises, la réflexion critique de Malevitch a refait le constat de l'écart entre l'expérience spatiale de l'homme et les structures de représentation "cunéiformes», issues de la perspective euclidienne. ${ }^{5}$ Le "Monde sans objet» rejetait essentiellement l'élément de base engendré par la perspective euclidienne, soit la notion d'un objet substantiel, autonome, volumétrique et isolable, que l'on avait coutume d'associer à celle d'objet «naturel».

De son côté, la réflexion formaliste du Futurisme avait proclamé la nécessité de l'éclatement des formes-contour dans l'objet représenté, si l'on veut rendre compte de l'expérience de nouvelles interrelations spatiales, notamment des modifications "physiques» subies par les objets-enmouvement - seuls objets concevables dans un univers post-einsteinien et des transformations «psychiques» du temps et de l'espace révélées par la révolution freudienne.

Cependant Malevitch lui-même se préoccupa davantage de l'analyse du «plan-plane», comme de l'élément additionnel qu'il apportait à l'analyse de l'objet pictural post-cubiste; que de la description des nouvelles structures spatiales qu'il instaurait dans la totalité de l'ouvre.

C'est dans l'oeuvre de Mondrian que l'on verra pour la première fois, interreliée de façon essentielle, la saisie de la fonction dynamique de nouveaux éléments picturaux au sein de structures spatiales différèntes du champ pictural global. Dès 1918, Mondrian substituait à la notion de l'objet naturaliste/volumétrique celle d'un nouvel «objet plastique», susceptible de représenter la relation dynamique entre les expériences interne et externe et obtenu par la «détermination» de la couleur et la position/dimension des

4. TARABOUKINE, Nikolai, «Pour une théorie de la peinture», le Dernier Tableau, Paris, Champ Libre, 1972, p. 132.

5. MALEVITCH, Casimir, "On New Systems in Art» (1919) Essays on Art (1915-1933), New York, G. Wittenborn, p. 113. 
plans de couleur. ${ }^{6}$ Mondrian rejetait avec force toute lecture statique et macroscopique de ses «lignes», toute identification de ses «rectangles» à des formes géométriques fermées, comme le voudrait la géométrie euclidienne :

Dans la forme apparente du rectangle, les lignes se coupent, se touchent en tangentes, mais ne cessent pas de continuer.

Il ne s'agit plus :

...de plans rectangulaires l'un à côté de l'autre comme des pierres. Le plan rectangulaire doit plutôt être considéré comme résultant de la pluralité de la ligne droite en opposition rectangulaire. ${ }^{7}$

Par la valorisation de la forme ouverte et des énergies périphériques, par l'élaboration d'éléments plastiques insérés dans des relations énergétiques contradictoires, Mondrian institue la dialectique fondamentale de la virtualisation ou potentialisation du champ spatial, qui rend possible la représentation d'un «plenum» spatial non-euclidien.

Mais en dépit de quelques réserves verbales, le discours de la critique formaliste réimpose sans cesse le modèle spatial euclidien dans le décodage d'une pseudo-grille uniforme dans l'œuvre de Mondrian, au lieu de percevoir les fonctions pluri-valentes des «séries de grilles». ${ }^{8} \mathrm{Ou}$ encore il conclut à «une représentation tronquée d'un objet dans un espace tridimensionnel», là où il faudrait faire appel à la représentation d'un espace non-euclidien, proche de la géométrie projective. ${ }^{9}$

De façon générale, la fixation persistante d'une valeur esthétique au simple mimétisme de l'objet référentiel, «tri-dimensionnel» ou volumétrique, a poussé la critique à noter progressivement la démarche déconstructrice de cet «objet» par les artistes post-cubistes, plutôt qu'à réfléchir sur les structures particulières de la notion d'espace qui avait rendu son émergence picturale possible et la structure des nouveaux espaces qui exigeaient sa disparition. Quand elle généralise à toute la surface du tableau la notion de "planéité» du plan de Malevitch pour qualifier tout l'ensemble de bidimensionnel, la critique formaliste américaine des années 50 fonde cette extension, non sur la théorie picturale, mais sur un.emprunt à la théorie de l'information, qui a pris son essor aux États-Unis quelques années plus tôt.

C'est en effet à partir d'une caractéristique du «support physique» ou du «canal de transmission» du message pictural, soit la planéité de la

6. MONDRIAN, Piet, "The Determinate and the Indeterminate», De Stijl, vol. II, 1918, pp. 14-19.

7. MONDRIAN, Piet, «L'art réaliste et l'art superréaliste», Cercle et Carré, 2, 15 avril 1930.

8. KRAUSS, Rosalind, «Grids», October, nº 9, été 1979, pp. 51-64.

9. SCHAPIRO, Meyer, «Mondrian, Order and Randomness in Abstract Painting", Modern Art, 19th and 20th Century, New York, G. Braziller, 1979, p. 235. 
surface de la toile, que l'on a voulu dégager les notions qui définiraient l'art moderniste. Car si la critique formaliste américaine a aussi attiré l'attention sur une autre caractéristique de ce support physique, soit la dynamique propre au format ou à la "délimitation de la surface», elle en a peu analysé l'effet dialectique, sauf pour les décuplements ou affaiblissements énergétiques produits par la répétition interne ou la contradiction de ses composantes les plus apparentes : l'horizontalité et la verticalité.

En un sens, on peut poser que Greenberg a interprété la fonction structurante du support physique qui véhicule le message pictural d'une façon encore plus restrictive que ne l'a fait Marshall McLuhan. Le spectaculaire aphorisme de celui-ci : "The Medium is the message», qui pose que la structure énergétique du canal de transmission constitue et détermine le "contenu» du message, s'accompagnait d'une analyse plus exhaustive et dynamique des structures propres à tout «medium» physique.

La notion de "planéité de la surface» définie par Greenberg a été utilisée de façon plus normative et incantatoire qu'analytique :

La qualité d'être plat, la bi-dimensionnalité est la seule chose que la peinture ne partage avec aucun autre art et c'est ainsi que la peinture moderniste s'oriente vers la planéité plus que vers toute autre chose. ${ }^{10}$

L'on pourrait certes se demander en quoi d'autres arts, tels le texte poétique, la photographie ou la pellicule cinématographique posséderaient moins cette qualité du support, "d'être plat».

Cette tentative de fonder les caractéristiques du phénomène pictural sur la simple description du support physique dans lequel s'ancre phénoménologiquement la transmission du message pictural, néglige des aspects encore plus fondamentaux de la constitution des messages, soit l'utilisation de signes particuliers à l'intérieur d'un certain code. Seul le code, comme système modélisant, permet de saisir la nature des éléments physiques dans leur fonction de «signifiants». Structure intermédiaire entre une intentionalité artistique et les moyens de transmission, le code investit les éléments sensibles, aussi bien que le support physique du message, d'une dimension sémantique particulière. Mais le code pictural aura toujours à structurer une construction spatiale, pour autant que l'espace se définit comme une coexis tence simultanée d'éléments multiples.

Ce modèle spatialisant qui doit fonder le code sémiologique de la peinture s'élabore au confluent de la psychologie et des seuls discours sur l'espace qu'offrent les géométries. De même que le recours à la linguistique génétique a paru nécessaire à W.O. Quine pour décrire le fonctionnement sémiologique/sémantique du langage verbal, ${ }^{11}$ il paraît indispensable

10. GREENBERG, Clement, «Modernist Painting», The New Art, New York, Dutton, 1969, pp. 360-371.

11. Cf. QUINE, W.O., «Relativite de l'ontologie et Autres Essais» (1969) Paris, Aubier, 1977. 
d'éclairer les sources génétiques de la notion d'espace pour décrire les divers systèmes de représentation spatialisée qu'offre l'art au $\mathrm{XX}^{\mathrm{e}}$ siècle.

La démarche épistémologique de Jean Piaget s'articule en deux moments tout à fait distincts. Le premier éclaire le champ vécu de l'expérience humaine, qui constitue le lieu référentiel de toute sémantique. À ce niveau, les expérimentations qu'il a menées révèlent que la notion d'espace est plurielle, c'est-à-dire que l'expérience concrète de l'homme élabore divers types d'espaces, hétérogènes les uns aux autres et qui s'unifient selon les potentialités de leurs éléments constituants. Le deuxième moment de cette démarche définit les bases d'un système syntaxique qui relie signifiants et signifiés dans divers modes de représentation de ces espaces. ${ }^{12}$

Piaget dévoile en premier lieu que dans son trajet existentiel d'appréhension et de "construction du réel», l'organisme humain élabore une multiplicité d' «espaces organiques» ou "pratiques», qui coordonnent ses activités sensori-motrices dans des schémas totalisants très diversifiés. Ces espaces organiques sont distincts entre eux et sont polarisés par les divers modes d'accès sensoriels de l'organisme au monde ambiant. Ce sont les espaces gustatif ou buccal, postural, thermique, auditif, visuel, tactile, kinesthésique ou sensori-moteur. En organisant les aspects qualitatifs/ quantitatifs prélevés perceptuellement dans la multiplicité du réel, selon des structures nécessairement différentes, ces «espaces» vécus retiennent du réel des éléments-en-relation qui constituent les corrélatifs fondamentaux auxquels renverra ultérieurement toute représentation spatiale. Or les structures d'organisation, de mise en relation des éléments dans les espaces vécus, organiques, sont d'ordre topologique.

Si l'étude des structures de ces espaces organiques existentiels peut se réaliser par une observation du comportement de l'enfant, l'analyse des modes de représentation qu'il en élabore est encore plus accessible à partir des constructions graphiques qu'il produit très tôt. Mais on ne saurait assez souligner la différence de statut qui existe entre l'expérience par laquelle l'organisme humain structure un type d'espace dans sa relation concrète au monde et les divers modes de représentation graphique, pictural ou sculptural, par lesquels il tente de se les représenter.

Pour s'élaborer, ces divers espaces organiques dépendent aussi bien du développement des activités sensori-motrices de l'organisme que du développement de ses facultés intellectuelles. C'est-à-dire que l'espace, ou les espaces, ne se présentent à aucun moment comme une donnée ou un

12. PIAGET, Jean, la Construction du réel, Genève, Delachaux \& Niestlé, s.d. PIAGET, Jean, la Représentation de l'espace chez l'enfant, Paris, P.U.F. 1948. 
«objet» offert à une impression sensorielle, mais plutôt comme une construction perceptuelle résultant de l'interaction entre les facultés sensorimotrices et les facultés intellectuelles, en vue d'unifier de quelque façon une multiplicité hétérogène :

L'espace est donc l'activité même de l'intelligence en tant qu'elle coordonne les tableaux extérieurs les uns aux autres. ${ }^{13}$

Ou encore:

L'intuition de l'espacè n'est pas une lecture des propriétés des objets, mais bien dès le début, une action exercée sur eux. ${ }^{14}$

Cette action exercée par l'organisme humain sur les existants dépend cependant de la structure des éléments sensoriels prélevés et de leur potentialité d'interrelations, engendrant par là des espaces sensoriels divers. Et c'est l'activité - même d'uassimilation-accomodation» par laquelle s'établissent les liens avec le réel qui constitue, selon Piaget, le «contenu référentiel» des premières tentatives de représentation du monde que fait l'enfant.

C'est-à-dire que le dessin de l'enfant ne prolonge pas une activité de "perception" pure, mais plutôt l'ensemble des mouvements, anticipations et reconstructions, comparaisons, qui constitue l'activité perceptrice. ${ }^{15}$

Même si les pulsions émotives en forment une composante, le processus de représentation chez l'enfant s'appuie sur. une volonté, un besoin, d'exprimer les conduites sensori-motrices qui ont mené à l'appréhension de ces formes/objets.

Les réalités visées ou intuitionnées dans la représentation graphique sont donc essentiellement les activités mêmes d'appréhension dư réel, qui sont «signifiées», relayées par l'image et non pas les images elles-mêmes dans leur fonction mimétique des objets. ${ }^{16}$

Sémiologiquement, ce n'est donc pas dans la dimension imagique, iconique, de l'image mimétique ou figurative que résident le signifié, le référent, le corrélatif existentiel du dessin, mais dans la possibilité de cette image de renvoyer aux ensembles spatiaux au sein desquels des systèmes d'action se sont déroulés dans l'expérience de la réalité. Plus encore, l'iconique, la représentation imagière, fondée sur une similarité statique,est toujours particulièrement impropre à évoquer, à reconstituer les transformations actives vécues par le sujet dans le monde, échouant même à évoquer les résultats terminaux de ces processus pulsionnels et moteurs constituant l'expériènce du réel:

13. PIAGET, Jean, la Construction du réel, Ibid., p. 185.

14. PIAGET, Jean, la Représentation de... Ibid., p. 532, p. 549.

15. PIAGET, Jean, la Construction du réel, Ibid., p. 185.

16. PIAGET, Jean, la Représentation de... Ibid., p. 541. 
Très tôt, cette image iconique devient pour l'enfant un obstacle majeur dans la tentative de reconstruction mnémonique des conduites qu'il a menées dans la réalité, tentative qui constitue le pôle même de l'acte de représentation. Dès l'âge de 12 ans, l'enfant éprouvera de la valorisation de cette image «iconique», qui ne sert que des conduites pragmatiques simples, un obstacle majeur au développement d'une pensée vraiment opératoire; permettant une adaptation plus complexe aux structures du réel:

Pour évoquer ses expériences et conduites antérieures dans une organisation d'éléments graphiques, l'enfant disposera des mêmés structures de regroupement des signes qu'il avait utilisées en construisant des espacés organiques au sein de la multiplicité du réel. Ce sont les ráppórts topologiques qui, par leur expressivité fonctionnelle, constituent des systèmes modélisants à la fois amples et précis, établissant des relations spatiales qui ne relèvent plus de la géométrie euclidienne. L'ensemble de ces rapports, sur un mode plus complexe et conscient, constitue les fondements du développement de l'art dit abstrait, depuis le début du XX $\mathrm{XX}^{\mathrm{e}}$, siècle, qu'il conviendrait davantage d'appeler art concret.

Ces rapports topologiques offrent cette singularité de' se présenter comme structures du signifiant et comme structures du signifié (ou du référent). À partir de là, le langage pictural ou sculptural s'offre à une prise plus directe de sa dimension sémantique que ne peut le faire le langage verbal, dont les structures syntaxiques prédicatives et leur déroulement linéaire ne peuvent qu'obscurcir la nature de la référence qu'ils véhiculent. Comme le notait justement Noam Chomsky, au sujet de la-syntaxe verbale, une véritable opération de transformation doit être effectuée pour déterminer sous la structure de surface linéaire le déploiement spatial des structures profondes :

Ainsi l'organisation sous-jacente d'une phrase, sujette à une interprétation sémantique, ne se révèle pas nécessairement dans l'agencemeńt effectif et le tour donné aux parties qui la composent.

Et corrélativement :

La structure de surface... n'exprime pas directement les rapports de signification existant entre les mots. ${ }^{17}$

Une difficulté accrue subsisterait dans la sémiologie du langage visuel, si l'on s'obstinait, comme l'a fait la critique formaliste américaine, à n'élaborer que des structures de surface, par analogie avec celles qu'a trop longtemps utilisées la linguistique verbale. Déjà Greimas a ressenti le besoin de faire appel à des modèles syntaxiques topologiques pour sortir à la fois de l'impasse sémantique créée par la structure linéaire du signifiant verbal et celle engendrée par la théorie du signe. ${ }^{18}$

17. CHOMSKY, Noam, la Linguistique cartésienne (1966), Paris, Editions du Seuil, 1969, p. 62.

18. GREIMAS, A.G., Sémantique structurale, Paris, Larousse, 1966, pp. 121-122. 
Ayant pris une extension considérable dans plusieurs secteurs des mathématiques actuelles, la topologie y est décrite à son premier niveau, comme une sorte de géométrie fruste, primitive, rudimentaire, sur laquelle se construisent toutes les autres géométries, euclidiennes ou non. La topologie développe les propriétés de l'espace qui demeurent invariantes à l'égard de déformations continues, c'est-à-dire des déformations qui excluent toute déchirure ou recouvrement; en d'autres mots, c'est la section des mathématiques qui s'occupe de la "continuité». ${ }^{19}$ Ainsi la topologie étudie un espace conçu comme un ensemble véritablement "continu» et homogène, où ne peuvent surgir des «trous» ou des "vides", au sein des éléments ou dans leurs interrelations.

La continuité de l'espace topologique résulte du caractère d'élasticité ou d'extensibilité dont sont dotés ses éléments, qui leur permettent de réagir qualitativement et quantitativement dans les trois dimensions sous l'action dynamique des forces environnantes. Sous l'action de l'environnement, ces éléments peuvent subir des transformations décisives de leurs caractéristiques préalables de forme, de couleur, de vectorialité, de tensions internes et de position dans la profondeur. La structure syntaxique de l'espace topologique est donc liée à la nature des éléments susceptibles de le constituer et inversement, la structure interne des éléments spatialisants entraîne une construction spatiale spécifique.

Les rapports topologiques qui fondent toute expérience de l'espace et, par suite, sa représentation plastique, sont d'ordre intensif et non métrique, fondés sur le voisinage et la séparation, l'entourage, l'emboîtement et l'enveloppement, la relation d'ordre ou de succession, la limite et la latéralité, l'ouverture et la fermeture, le joint et le disjoint, l'intérieur et l'extérieur, les points d'accumulation ou masse critique, etc., créant essentiellement une profondeur dans le continu.

Tout en définissant l'espace topologique, la notion de "continu» constitue, selon Piaget, la condition sine qua non de la construction de tout espace concret. Selon lui, en effet, toute autre forme de regroupement d'éléments discontinus constitue plutôt des ensembles ou des classes logiques, qui ne relèvent plus du phénomène de spatialisation :

Un espace est, en effet, un schème unique, englobant en un bloc d'un seul tenant tous les éléments qui le composent, tandis qu'une classe logique est un ensemble d'éléments discontinus, réunis par leurs seules ressemblances indépendamment de leurs distances dans l'espace et le temps. ${ }^{20}$

19. SAUVY, Jean et Simonne, la Découverte de l'espace chez l'enfant, Paris, Casterman, 1972, pp. 33-35.

20. PIAGET, Jean, la Représentation de..., Ibid., p. 541. 
C'est-à-dire qu' «un continu logique» n'est pas l'équivalent d'un espace ou d'un continu spatial. Un espace «formel» ne peut constituer un espace que s'il maintient de façon essentielle la fonction du continu entre ses éléments.

C'est l'intrusion de la notion logique de «points de vue» différents et discontinus, soit de visées spatiales dont le sujet n'a pas une expérience propre, qui entraînera au sein des modes de représentation l'émergence des espaces projectifs. Bien qu'elle se construise à partir de rapports topologiques, la représentation élaborée par les espaces projectifs intercale des points de rupture dans le tissu spatial, analogue à certains égards à ceux qu'étudie la théorie des catastrophes, soit l'émergence de charges énergétiques qui ne peuvent s'écouler de façon continue dans le milieu ambiant, mais qui sont abruptement transformées en qualités vectorielles non assimilables dans une spatialité du continu. ${ }^{21}$

Cependant si l'art enfantin n'élabore un espace projectif qu'à partir des premières intuitions de la ligne droite perspectiviste pour aboutir à la représentation euclidienne, l'art pictural depuis Cézanne a entrepris le trajet inverse. Les développements de la géométrie projective non-euclidienne incorporant l'action périphérique de points de vue "à partir de l'infini» peuvent rendre compte de certains espaces des Constructivistes ou de certaines ouvres de Mondrian. Ils correspondent de façon immédiate à un rejet du mode de représentation euclidien, ressenti comme un modèle logique d'éléments disjoints et discontinus, régis par des rapports aprioriques de transformation et fondés sur la métrique et l'émergence du «vide».

Conçue en effet pour rendre compte de «tous les points de vue possibles» sur un objet, la perspective euclidienne a dû sacrifier à cette fonction logique, la capacité de rendre compte de l'intuition spatialisante elle-même. Elle interprète la notion qualitative de «séparation» topologique, en y incorporant une dimension structurelle et quantitative du «vide», qui tient à distance les éléments entre eux, dans une localisation immuable et qui fige leurs contours, leurs formes et leurs couleurs dans des rapports de proportion fixes.

L'affermissement du «vide euclidien» entre les objets, qui tient en échec leurs interrelations possibles, se symbolise essentiellement dans la notion de volumétrie stable des objets, imaginable dans la seule hypothèse d'une discontinuité et d'une non-réaction du milieu ambiant à l'émergence des objets, soit l'hypothèse même d'un vide spatial qui environnerait les objets et dans lequel ils se logeraient. Mais, comme le souligne Piaget, les intervalles de vide entre les objets trop séparés, n'appartiennent pas à l'espace ou aux mêmes espaces que les éléments pleins. ${ }^{22} \mathrm{De}$ fait, ils

21. Cf. THOM, René, Stabilité structurelle et morphogénèse, Mass., W. Benjamin Inc., 1972.

22. PIAGET, Jean, la Représentation de..., Ibid., p. 554. 
n'appartiennent pas à l'espace comme corrélatifs d'une expérience sensible, mais résultent d'une opération logique tardive, introduisant la dialectique du discontinu, d'une contradiction spécifique entre le plein et le vide, laquelle, si intéressante qu'elle soit sur le plan logique, ne relève plus de l'intuition de l'espace lui-même.

Les contradictions de cette perspective, fondée sur la pseudohomogénéité d'un espace vide meublé par des objets pleins, sont rapidement apparues à la Renaissance, alors qu'il a fallu adjoindre une série de déterminations contraignantes pour lier explicitement des éléments qui demeurent discontinus et pour faciliter la représentation d'une distance très lointaine. À la perspective linéaire menant au point de fuite sur la ligne d'horizon, il fallut ajouter les calculs des points de distance latéraux, la perspective atmosphérique, le calcul des raccourcis, l'unicité de la source d'éclairage, la distribution des clairs-obscurs, la superposition des contours, etc. En particulier, cette hypothèse perspectiviste axée sur le point de fuite à l'infini a mené à une confusion abusive sur la notion même de profondeur, identifiée à une grande distance dans le lointain. Elle occultait, pour des raisons idéologiques faciles à déterminer, l'expérience de la profondeur proxémique, cette profondeur du proche où se relient le moi et le non-moi, région par excellence de l'expérience humaine, concrète et émotive. Ce sont à ces nouvelles dimensions proxémiques que renvoient les nouveaux espaces picturaux au XXe siècle depuis le Cubisme, chez Malevitch et Mondrian, chez Pollock, Dubuffet, Borduas et Molinari. C'est la profondeur structurelle des espaces topologiques.

Ainsi les qualités de mouvance proxémique des surfaces topologiques permettent de clarifier la notion de "planéité de la surface» que la critique formaliste américaine a voulu identifier à une hypothétique et utopique bidimensionnalité de la surface fictionnelle de la peinture, détruisant ainsi la fonction de spatialisation de la représentation picturale. Les rapports topologiques permettent, au contraire, de comprendre cette planéité comme une profondeur proxémique non-euclidienne, où les signifiants (colorèmes, vecteurs, etc.) se disposent dans «l'avant» et «l'arrière» dans une ondulation continue tri-dimensionnelle, qui renvoie aux espaces perceptuels organiques.

Cette voie épistémologique permet d'élaborer une sémiologie du langage visuel où la syntaxe des éléments signifiants peut se relier étroitement à la structure des réseaux sémantiques qui lui sont liés. Si le sémantique visuel doit découler, selon la définition de Michel Serres, d'une position dans un espace qualifié, il logera aussi dans les morphologies spatiales que seule la topologie peut articuler. Cela nous permet donc de conclure avec Michel Serres :

Et donc, premièrement, la topologie est une esthétique. Et donc, deuxièmement, une sémiologie. On n'en finit jamais avec l'espace. On 
ÉTUDES 95

ne parle jamais que de lui et en lui. Jamais on ne le quitte. Pour aller où, je vous le demande. 23

23. SERRES, Michel, Esthétiques sur Carpaccio, Paris, Hermann, coll. Savoir, 1975, p. 85. 